\title{
Fate of Entanglement for Initial Separable States in Quantum Neural Network
}

\author{
U. KORKMAZ
}

\begin{abstract}
This study is related to the fate of entanglement for initial separable states in a quantum neural network (QNN) model, which is in contact with the data environments locally. The duration of entanglement in quantum systems becomes extremely important when we consider it as a valuable resource. Thus, the effects of various initial states on the occurrence or decay of entanglement are investigated in the presence of information reservoirs. Especially in this study, central spin model has been examined as a quantum version of neural networks by taking inspiration from the biological models. Our model consists of a central spin system with two nodes to which the nodes are coupled to independent spin baths. Numerical results clearly show that different initial states have a profound effect on the fate of the entanglement. It also shows that the entanglement lifetime can be adjusted by regulating the reservoir states. The results can be used in realistic communication network situations to improve the performance of entanglement formation or distribution.
\end{abstract}

Index Terms - Entanglement, Information reservoir, Central spin model, Quantum neural network.

\section{INTRODUCTION}

$\mathrm{I}^{\mathrm{N}}$ $\mathrm{N}$ RECENT studies in the field of artificial intelligence, especially machine learning and artificial neural networks (ANN) have become popular. Machine learning, a field of computer science at the beginning of ANN, provides the ability to learn without explicit programming to computers [1-3]. Neural networks, which are interconnected computing structures based on binary McCulloch-Pitts neurons, are inspired by biological foundations [2]. The Hebb's learning rule, based on biological and neurophysiological basis, aims to obtain the best learning by changing the weight of the relevant units [4]. When classical learning rules address the dynamics in a statistical information environment, they manage them in the form of probability density functions [5]. Thus, the formulations and constraints of learning laws are based on the relationships between the global and local information environments of each transaction item.

UFUK KORKMAZ, is with Department of Electrical Engineering University of Istanbul Technical University, Istanbul, Turkey,(e-mail: ufukkorkmaz@itu.edu.tr).

iD https://orcid.org/0000-0001-5836-5262

Manuscript received January 12, 2019; accepted April 26, 2019.

DOI: $\underline{10.17694 / \text { bajece. } 541444}$
Networks that contain quantum material are known as quantum networks, which are important for the distribution of quantum resources and quantum communication [6]. The use of these quantum networks as neural networks has triggered several studies, including attempts to implement QNN suggestions in the context of quantum computations [7-18]. In this respect, quantum systems make the applications more attractive than classical communication tasks. In particular, one of the most interesting features of quantum mechanics is the entanglement. [19-21]. For this reason, it is important to understand their properties and dynamics for a number of applications in quantum mechanics. There are also many areas where entanglement plays a central role. For example, the transmission of unknown quantum data, quantum cryptography, secure communication, quantum measurement and quantum information processing [20-24]. In spite of these effective applications, because of the fragile nature of the entanglement, it is an important challenge to maintain and distribute entanglement for a sufficient amount of time.

When entangled system units come into contact with environmental degrees of freedom, entanglement decays to zero within a finite period. This is referred to as entanglement sudden death (ESD) [20, 25-27]. It was also observed experimentally by Almeida et al [28]. The entanglement dynamics in the common and independent reservoirs vary dramatically depending on the initial conditions of the system in the Marko vian information environment [21]. In some cases, entanglement can be lost for a limited period of time, and then can revive again. The reason for this is that the common reservoirs tend to create entanglement instead of destroying completely it as a result of combining the qubits indirectly. Due to the correlation created by the environment, even if qubits are initially prepared in a factorized condition, may lead to a phenomenon known as entanglement sudden birth (ESB) [20, 29].

In this study, entanglement dynamics are investigated after creating a simple QNN architecture [30]. Although the dynamic behavior of quantum entanglement under certain assumptions in QNN architecture has been well studied for a few qubits scales, it should be reassessed for the sake of using quantum resources. Moreover, due to the rapid advances in technology, the entanglement production has been achieved more rapidly than the entanglement decay [31]. In particular, we investigate the dynamics of a small QNN unit in contact with the quantum information reservoir. In the Ref. [21], the information store concept was initially introduced in a classical style and was studied for quantum systems. We simulate the proposed quantum system up to three input nodes with different states 
and examine the open system dynamics. This study relates to the numerical simulation of the dynamic evolution of an open QNN that is initially separated. The basic assumption of this paper is that QNNs in information environments are considered as open quantum systems. Therefore, the purpose of the study is to examine the dynamics of entanglement based on the quantum data of QNNs. Open system dynamics are modeled by repeated interactions $[32,33]$ between the local nodes of the network and the units representing the reservoir. These repeated types of interactions have been shown to be dynamic maps equivalent to the Markovian principal equation approach, using the divisibility of quantum channels [34]. We have found that the initial states of the quantum network are strongly related to the entanglement dynamics. Both asymptotic decay and the decay of entanglement in finite time have been observed due to diversity in environmental states.

This paper is organized as follows: In Sec. II, we present the physical model and system dynamics. In Sec. III, we analyze the QNN as a system that interacts weakly with the information reservoir in the Markov approach. Finally, we conclude this work in Sec. IV.

\section{PHYSICAL MODEL AND SYSTEM DYNAMICS}

One of the most important factors in the emergence of neural networks is the studies to perform advanced cognitive tasks [5]. Perceptron is the most simple neural network unit. And inspired by perceptron, we created the QNN model as equivalent to the central spin model (also known as the spinstar network) which has with various applications such as the decay of quantum coherence [35] and quantum communication [36]. In Ref. [37], the dynamic evolution of central spin quantum consistency based on different spin binding types with Markov Dynamics was investigated. It has also been shown that this model may have advantages on the quantum scale for thermodynamic studies [38]. In this study, the central spin model inspired by biological models (Fig. 1 (a)) was examined as a quantum version of neural networks.

The system of interest is treated as an open quantum system and consists of three interactive qubits with a flip-flop type Hamiltonian. The time-independent Hamiltonian representing system dynamics can also be used for quantum effects in biological systems [39]. This is expressed as follows

$$
\begin{aligned}
H= & \frac{\omega}{2}\left(\sum_{i}^{N} \sigma_{i}^{Z}+\sum_{i=1}^{N-1} \sigma_{u_{i}}^{Z}\right)+\left(\sum_{i=1}^{N-1} J_{i} \sigma_{i}^{+} \sigma_{\text {out }}^{-}+\right. \\
& \left.\sum_{i=1}^{N-1} J_{i} \sigma_{u_{i}}^{+} \sigma_{i}^{-}+\text {H.c. }\right)
\end{aligned}
$$

where $\omega, \sigma^{z}, \sigma^{+}, \sigma^{-}$and,$\sigma_{\text {out }}^{-}$are Bohr frequency each spin and single qubit information unit has been taken equal for simplicity, Pauli-z, raising and lowering operators for the qubits and Pauli operator for output qubit, respectively. Also, $J_{i}$ is the coupling coefficient between input units and the output node, $\sigma_{i}$ is the Pauli operator representing an individual node in contact with the information reservoir and $\sigma_{u_{i}}$ is the Pauli operator of the individual unit representing the information reservoir.

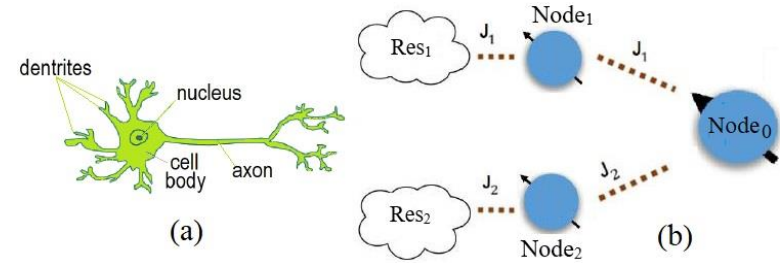

Fig.1. In (a) a biological neuron. In (b) unitary dynamics of a QNN unit with two input nodes

In this study, we use density matrix formalism to represent the quantum states of the relevant system. The quantum neurons are initially assumed to be in a product state as $\rho(0)=\rho_{\text {Res }} \otimes$ $\rho_{\text {Sys }}(0)$, where $\rho_{\text {Res }}=\rho_{\text {Res } 1} \otimes \rho_{\text {Res2 }}$ and $\rho_{\mathrm{s}}(0)=\rho_{1}(0) \otimes$ $\rho_{2}(0) \otimes \rho_{\text {out }}(0)$ are Reservoir units and System units, respectively. Individual qubit states were chosen as $\rho(0)=$ $|\uparrow\rangle\langle\uparrow|$ in order to initially provide a blank memory. Here, $\rho_{\text {up }}=$ $|\uparrow\rangle\left\langle\uparrow\left|, \rho_{d n}=\right| \downarrow\right\rangle\langle\downarrow|,| \mp\rangle=\frac{1}{\sqrt{2}}((|\uparrow\rangle \mp|\downarrow\rangle))$ are the cat states where $|\uparrow\rangle,\langle\downarrow|$ are orthogonal spin states known as computational basis in quantum computing language.

Open system dynamics has been discussed by adopting a repetitive interaction process, also known as a collision model [40]. Fig. 1 (b) represents the open dynamics of the network to illustrate the specific use of the collision model. The initially and identically arranged qubit states that interact with the nodes of the neural network are obtained by tracing out the reservoir (environment) degrees of freedom as

$$
\rho_{s}(t+\tau)=\operatorname{Tr}_{u_{i}}\left[U(\tau) \rho_{s}(t) \otimes \rho_{u_{i}} U(t)^{\dagger}\right]
$$

where, $\operatorname{Tr}_{u_{i}}$ stands for partial trace over en vironmental degrees of freedom, $U(\tau)=e^{-i H \tau}$ is the unitary operator representing the system plus environment dynamics for time independent Hamiltonians and $\tau$ is the duration of each unit-node interaction. Calculations were made using exact diagonalization. In our model, $\rho_{S}$ the state of the system of interest (QNN) and $\rho_{\text {Res }}=\rho_{u_{i}}$ represents the quantum state of each single qubit environment unit.

The aim of our study is to examine the change in the entanglement according to various initial states. That's why we are investigating that there is no initial entangled between the readout node and one of the input nodes in contact with the environment. After the system interacts with the information reservoir, the environment needs to send information to the system so that the system can be balanced with the environment carrying the quantum information content. Concurrence is a scalar function. It is used to measure the entanglement of density matrices that initially define the mixed states of a system [41]. In this paper concurrence is accepted as a measure of entanglement and calculated as $C=\max \left[0, \lambda_{1}-\lambda_{2}-\lambda_{3}-\right.$ $\lambda_{4}$ ] where $\lambda_{i}$ are the square roots of non-Hermitian matrix $\rho \tilde{\rho}$, $\rho$ is the density matrix to be calculated and $\tilde{\rho}$ is its spin flipped from such as $\tilde{\rho}=\left(\sigma_{y} \otimes \sigma_{y}\right) \rho^{*}\left(\sigma_{y} \otimes \sigma_{y}\right)$ where $\rho^{*}$ is the complex conjugated from of $\rho$, the descending order [42].

\section{RESULTS}

We analyze QNN, which we consider as a system that interacts weakly with the information store in the Markov approach. Due to this approach, the temporal evolution of the system only depends on the current state of the system. That is, 
the quantum state of the system is lost irreversibly with reservoir degrees of freedom. In our scenario, each of the quantum information reservoirs interacts weakly with one of the input nodes (local subsystems) of the QNN unit. Pure single qubit states $(|\uparrow\rangle\langle\uparrow|$ and $|\downarrow\rangle\langle\downarrow|)$ can represent information environments. The initial quantum state of the en vironment plus system is represented by $\rho(0)=\rho_{\text {env }} \otimes \rho_{\text {sys }}(0)$ where $\rho_{\text {sys }}(0)=\rho_{1}(0) \otimes \rho_{2}(0) \otimes \rho_{0}(0)$. Here, $\rho_{1}, \rho_{2}$ are the quantum states of the input nodes $\left(\right.$ Node $\left._{1}, N_{\text {ode }}\right)$ and $\rho_{0}$ stands for the output node $\left(N_{o d e}\right)$. Also, quantum state of the environmental qubit states are $\rho_{\text {env }}=\rho_{\text {Res1 }} \otimes \rho_{\text {Res2 }}$ and fixed. Then, the concurrence time evolution between the two specific qubits of the three-qubit QNN system will be examined according to various initial states.

Fig. 2 represents the evolution of concurrence for initial separable states of the quantum neuron depending on the number of collisions (nc). In Fig. 2, the initial system plus environment states are a): $\rho(0)=\rho_{d n} \otimes \rho_{\text {up }}(0) \otimes \rho_{|+\rangle}(0) \otimes$ $\left.\rho_{\text {up }}(0) \otimes \rho_{\mathrm{dn}}, \mathbf{b}\right): \rho(0)=\rho_{d n} \otimes \rho_{\mathrm{up}}(0) \otimes \rho_{\mathrm{dn}}(0) \otimes$ $\left.\rho_{\text {up }}(0) \otimes \rho_{\mathrm{dn}}, \boldsymbol{c}\right): \rho(0)=\rho_{d n} \otimes \rho_{\mathrm{dn}}(0) \otimes \rho_{\mathrm{up}}(0) \otimes$ $\rho_{d n}(0) \otimes \rho_{\mathrm{dn}} \quad$ and $\left.\quad \mathbf{d}\right): \rho(0)=\rho_{u p} \otimes \rho_{\mathrm{up}}(0) \otimes \rho_{|+\rangle}(0) \otimes$ $\rho_{\text {up }}(0) \otimes \rho_{\mathrm{dn}}$. In Fig. 2 a), despite there is no initial entangled between the nodes of the QNN, during the early evolution of concurrence a rapid formation of entanglement between the nodes is evident. The observed entanglement rapidly decays as the system evolution continues (Fig. 2 a), b) (between $N_{\text {ode }}$ and $\left.\operatorname{Node}_{2}\left(C_{21}\right)\right)$ and $\left.\mathbf{d}\right)$ ). More interestingly in the later evolution, a sudden birth of entanglement between $N_{\text {ode }}$ and Node $_{2}\left(C_{21}\right)$ was observed where the nodes do not directly interact (Fig. 2 a) and b)). However, the revival scheme of Fig. 2 b) is slightly weaker than that observed in Fig. 2 a). In Fig 2 c), a rapid formation of entanglement in the very beginning of the evolution is appear for both $C_{20}$ and $C_{21}$ despite there is no initial entangled between the nodes of the QNN. As obvious they initial entangled decays exponentially and oscillotary. This applies to $C_{21}$ in Fig. 2 b). Especially in Fig. 2 d) initial entangled decays fast and end up in finite time [43].
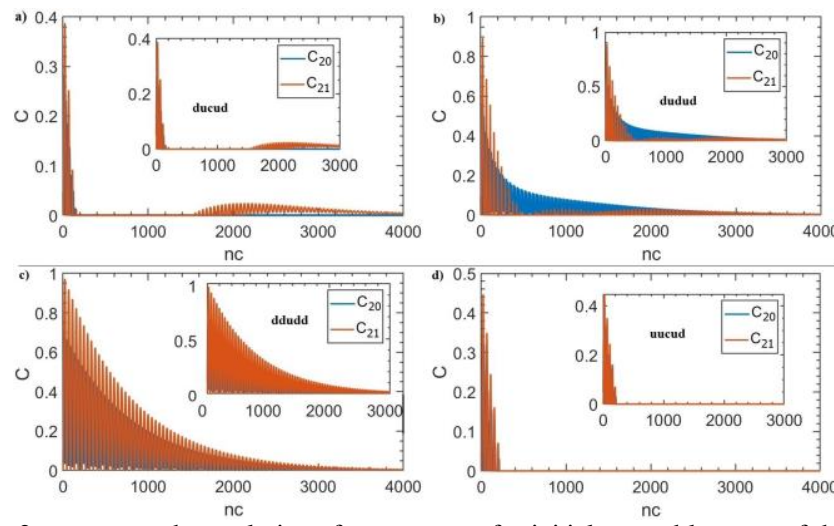

Fig.2. represents the evolution of concurrence for initial separable states of the quantum neuron depending on the number of collisions (nc).. a) The quantum state of the quantum neuron as $\operatorname{Node}_{1}=\rho_{\text {up }}, \operatorname{Node}_{2}=\rho_{\text {up }}$ and Node $e_{0}=\rho_{|+\rangle}$ states. The state of the units representing the environment were set as $\rho_{\mathrm{dn}}$ for both of them. b) The quantum state of the quantum neuron $\operatorname{Node}_{1}=\rho_{\text {up }}$, Node $_{2}=\rho_{u p}$ and Node $_{0}=\rho_{\text {|dn }}$ states. The state of the units representing the environment were set as $\rho_{\mathrm{dn}}$ for both of them. c) The quantum state of the quantum Node ${ }_{1}=\rho_{\mathrm{dn}}$, Node $_{2}=\rho_{d n}$ and Node ${ }_{0}=\rho_{\text {up }}$ states. The state of the units representing the environment were set as $\rho_{\mathrm{dn}}$ for both of them. d) The quantum state of the quantum neuron as Node $_{1}=\rho_{\text {up }}$, Node $_{2}=\rho_{\text {up }}$ and Node $_{0}=\rho_{|+\rangle}$states. The state of the units representing the environment were set as $\operatorname{Res}_{1}=\rho_{\text {up }}$ and $\operatorname{Res}_{2}=\rho_{d n}$. The coupling between the environment unit and the input node is equal to the coupling between the input and the readout node $J_{1}=J_{2}=J=0.1$. The duration of the each unit interaction $\tau$ between the units and the input node is $\tau=5 \times 10^{-2} / \mathrm{J}$

\section{CONCLUSION}

We have connected the QNN unit, which we consider as open system dynamics, to two reservoirs by the conventional flipflop Hamiltonian. We analyze QNN, which we consider as a system that interacts weakly with the information store in the Markov regimes. Each of the quantum information reservoirs interacts weakly with one of the input nodes (local subsystems) of the QNN unit. We adopt a collisional model to simulate open system quantum dynamics. The study also examines the generation of entanglement due to initially separable states. Numerical results clearly show that different initial states have a profound effect on the fate of the entanglement. We show that both entanglement revival (ESB) and entanglement sudden death (ESD) occur in the separable initial states. The results can be used to evolve the QNNs to distribute or connect the entanglement resource.

\section{REFERENCES}

[1] S. Russell, P. Norvig, Artificial Intelligence: A Modern Approach. USA: Pearson, Upper Saddle River, 2009.

[2] W.S. McCulloch, W. Pitts, "A logical calculus of the ideas immanent in nervous activity" The bulletin of mathematical biophysics, Vol.5, 4, 1943, pp.115-133.

[3] F. Rosenblatt, "The perceptron: A probabilistic model for information storage and organization in the brain" Psychological Review, Vol.65, 6, 1958, pp.386-408.

[4] D. O. Hebb, The Organization of Behavior: A Neuropsychological Theory, Psychology Press, Mahwah NJ, 2002.

[5] R. Hecht-Nielsen, Neurocomputing, Boston, MA, USA: AddisonWesley, 1990.

[6] J. I. Cirac, P. Zoller, H. J. Kimble, H. Mabuchi, "Quantum state transfer and entanglement distribution among distant nodes in a quantum network" Physical Review Letters, Vol.78, 16, 1997, p. 3221.

[7] M. Lewenstein, M. Olko, "Quantum neural networks" Network: Computation in Neural Systems, Vol.2, 1, 1991, pp.207-230.

[8] S. Kak, "On quantum neural computing" Information Sciences, Vol.83, 3-4, 1995, pp.143-160.

[9] M. Lewenstein, "Quantum Perceptrons" Journal of Modern Optics, Vol.41, 12, 1994, pp.2491-2501.

[10] I. E. Lagaris, A. Likas, D. I. Fotiadis, "Artificial neural network methods in quantum mechanics" Computer Physics Communications, Vol.104, 13, 1997, pp.1-14.

[11] M. Zak, C. P. Williams, "Quantum Neural Nets" International Journal of Theoretical Physics, Vol.37, 2, 1998, pp.651-684.

[12] A. Narayanan, T. Menneer, "Quantum artificial neural network architectures and components" Information Sciences, 2000; Vol.128, 34, 2000, pp.231-255.

[13] D. Ventura, T. Martinez, "Quantum associative memory" Information Sciences, 2000; Vol.124, 1-4, 2000, pp.273-296.

[14] S. Gupta, R. K. P. Zia, "Quantum Neural Networks” Journal of Computer and System Sciences, Vol.63, 3, 2001, pp.355-383.

[15] M. Panella, G. Martinelli, "Neural networks with quantum architecture and quantum learning" International Journal of Circuit Theory and Applications, Vol.39, 1, 2011, pp.61-77.

[16] R. Zhou, H. Wang, Q. Wu, Y. Shi, "Quantum Associative Neural Network with Nonlinear Search Algorithm" International Journal of Theoretical Physics, Vol.51, 3, 2012, pp.705-723.

[17] M. Schuld, I. Sinayskiy, F. Petruccione, "Simulating a perceptron on a quantum computer" Physics Letters A, 2015; Vol.379, 7, 2015, pp.660663. 
[18] L. Banchi, N. Pancotti, S. Bose, "Quantum gate learning in qubit networks: Toffoli gate without time-dependent control" npj Quantum Information, Vol.2, 2016, p.16019.

[19] R. Horodecki, P. Horodecki, M. Horodecki, K. Horodecki, "Quantum entanglement” Reviews of Modern Physics, Vol.81, 2, 2009, p.865.

[20] L. Mazzola, S. Maniscalco, J. Piilo, K. A. Suominen, B. M. Garraway, "Sudden death and sudden birth of entanglement in common structured reservoirs" Physical Review A, Vol.79, 4, 2009, p.042302.

[21] D. Türkpençe, "Disentanglement Dynamics of a Data Driven Quantum Neural Network" NeuroQuantology, Vol.16, 10, 2018, pp.14-19.

[22] C. H. Bennett, G. Brassard, C. Crépeau, R. Jozsa, A. Peres, W. K. Wootters, "Teleporting an unknown quantum state via dual classical and Einstein-Podolsky-Rosen channels" Physical Review Letters, 1993; Vol.70, 13, 1993, pp.1895-1899.

[23] V. Scarani, H. Bechmann-Pasquinucci, N. J. Cerf, M. Dušek, N Lütkenhaus, M. Peev, "The security of practical quantum key distribution" Reviews of Modern Physics, Vol.81, 3, 2009, p.1301.

[24] A. Galindo, M. A. Martin-Delgado, "Information and computation: Classical and quantum aspects" Reviews of Modern Physics, Vol.74, 2, 2002 , p.347.

[25] A. Al-Qasimi, D. F. V. James, "Sudden death of entanglement at finite temperature" Physical Review A, Vol.77, 1, 2008, p.012117.

[26] T. Yu, J. H. Eberly, "Sudden Death of Entanglement" Science, Vol.323, 5914, 2009, pp.598-601.

[27] J. León, C. Sabín, "Photon exchange and correlation transfer in atomatom entanglement Dynamics" Physical Review A, Vol.79, 1, 2009, p.012301.

[28] M. P. Almeida, F. de Melo, M. Hor-Meyll, A. Salles, S. P. Walborn, et al., "Environment-Induced Sudden Death of Entanglement" Science Vol.316, 5824, 2007, pp.579-582.

[29] Z. Ficek, R. Tanaś, "Delayed sudden birth of entanglement" Physical Review A, Vol.77, 5, 2008, p.054301.

[30] M. Schuld, I. Sinayskiy, F. Petruccione, "The quest for a quantum neural network" Quantum Information Processing, Vol.13, 11, 2014, pp.256786.

[31] P. C. Humphreys, N. Kalb, J. P. Morits, R. N. Schouten, R. F. Vermeulen, et al., "Deterministic delivery of remote entanglement on a quantum network" Nature, Vol.558, 7709, 2018, p.268.

[32] M. Ziman, P. Štelmachovič, V. Bužek, M. Hillery, V. Scarani, et al., "Diluting quantum information: An analysis of information transfer in system-reservoir interactions" Physical Review A, Vol.65, 4, 2002, p.042105.

[33] V. Scarani, M. Ziman, P. Štelmachovič, N. Gisin, V. Bužek, "Thermalizing Quantum Machines: Dissipation and Entanglement" Physical Review Letters, Vol.88, 9, 2002, p.097905.

[34] M. M. Wolf, J. I. Cirac, "Dividing quantum channels" Communications in Mathematical Physics, Vol.279, 1, 2008, pp.147-68.

[35] W. H. Zurek, "Decoherence, einselection, and the quantum origins of the classical" Reviews of Modern Physics, Vol.75, 3, 2003, p.715.

[36] Q. Chen, J. Cheng, K. L. Wang, J. Du, Optimal quantum cloning via spin networks" Physical Review A, Vol.74, 3, 2006, p.034303.

[37] D. Türkpençe, T. C. Akıncı, S. Şeker, "Decoherence in a quantumneural network" NeuroQuantology, Vol.16, 6, 2018, pp.1-5.

[38] D. Türkpençe, F. Altintas, M. Paternostro, O. E. Müstecaplıoğlu, "A photonic Carnot engine powered by a spin-star network" EPL (Europhysics Letters), Vol.117, 5, 2017, p.50002.

[39] E. J. O'Reilly, A. Olaya-Castro, 'Non-classicality of the molecular vibrations assisting exciton energy transfer at room temperature" Nature Communications, Vol.5, 3012, 2014.

[40] D. Türkpençe, T. C. Akıncı, S. Şeker, "A steady state quantum classifier" Physics Letters A, 2019; In Press. doi: 10.1016/j.physleta.2019.01.063

[41] R. Hildebranda, "Concurrence revisited" Journal of Mathematical Physics, Vol.48, 10, 2007, p.102108.

[42] S. Hill, W. K. Wootters, "Entanglement of a Pair of Quantum Bits" Physical Review Letters, Vol.78, 26, 1997, p.5022.

[43] T. Yu, J. H. Eberly, "Finite-time disentanglement via spontaneous emission" Physical Review Letters, Vol.93, 14, 2004, p.140404.

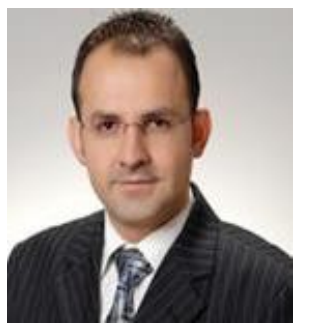

\section{BIOGRAPHIES}

UFUK KORKMAZ was born in Turkey. He received the BSc, MSc and $\mathrm{PhD}$ degrees from the Ondokuz Mayıs University (OMU), Physics Department, in 2006, 2010 and 2014 respectively. He starting researches as Post-Doc in Istanbul Technical University (ITU) in 2018. His research interests are IR and UV spectroscopy, X-ray single crystal diffraction, Understanding the nature of $\mathrm{H}$ bonds in supramolucular stracture, Quantum Mechanics and Quatum information theory. 